\title{
Cryptotanshinone-Loaded Cerasomes Formulation: In Vitro Drug Release, in Vivo Pharmacokinetics, and in Vivo Efficacy for Topical Therapy of Acne
}

Ting Zuo, ${ }^{\dagger, \|}$ Huoji Chen, ${ }^{\dagger, \|}$ Shijian Xiang, ${ }^{\dagger}$ Junhui Hong, ${ }^{\dagger}$ Siwei Cao, ${ }^{\dagger}$ Lidong Weng, $^{\dagger}$ Lu Zhang, $^{\dagger}$ Li Liu, ${ }^{\dagger}$ Hui Li, ${ }^{\ddagger}$ Hongxia Zhu, ${ }^{*} \S$ and Qiang Liu* ${ }^{* \dagger}$

${ }^{\dagger}$ School of Traditional Chinese Medicine, Southern Medical University, 1023 Shatainan Road, Guangzhou 510515, P. R. China

${ }^{\ddagger}$ Guangzhou Red Cross Hospital, 396 Tongfuzhong Road, Guangzhou 510220, P. R. China

${ }^{\S}$ Combining Traditional Chinese and Western Medicine Hospital, Southern Medical University, 13 Shiliugang Road, Guangzhou 510315, P. R. China

\section{Supporting Information}

ABSTRACT: Cerasomes (CS), evolved from liposomes, are novel drug-delivery systems that have potential medical application as carriers for drugs or active ingredients. Although many studies have been conducted on the pharmaceutical and physicochemical properties of CS, the role of CS in influencing the in vivo plasma and topical pharmacokinetics and efficacy of topical drug delivery remain unclear. In this context, we chose cryptotanshinone (CTS) as a model drug for the preparation of CTS-CS by means of the ethanol injection method to investigate their in vitro/in vivo drug-release behavior and in vivo efficacy. (1) In in vitro studies, CTS-CS gel was proven to be capable of achieving a higher permeation rate and significant accumulation in the dermis of isolated rat skin using Franz diffusion cells. (2) In in vivo studies, microdialysis experiments used to measure the plasma and topical pharmacokinetics demonstrated that the CS had a high drug concentration, short peak time, and slow elimination. Meanwhile, the plasma area under the concentration-time curve of CTS-CS gel was less than half that for the CTS gel in $12 \mathrm{~h}$, which indicates that the drug bioavailability dramatically increased in the experiments. (3) In in vivo efficacy studies, we duplicated a rat acne model and performed antiacne efficacy experiments. The CTS-CS gel improved the antiacne efficacy compared to that of ordinary CTS gel. Moreover, it inhibited the expression of interleukin-1 $\alpha$ and androgen receptors effectively. All of these results show that CTS-CS gel has significant potential for the treatment of acne induced by inflammation and excessive secretion of androgen, suggesting that CS formulations were designed as a good therapeutic option for skin disease.

\section{INTRODUCTION}

Cerasomes (CS), mainly consisting of ceramide, phospholipid, cholesterol, and other components, are a new type of drugdelivery system evolved from liposomes, which have a low toxicity and are highly biocompatible. ${ }^{1-5}$ As compared with conventional liposomes, CS, when applied externally, tend to dissolve and penetrate into the stratum corneum (SC) cells because of their high affinity for keratin. ${ }^{6,7}$ When encapsulating drugs or active ingredients as a carriers, CS can improve the stability of the inclusions. Besides, hair-follicle-targeting provides the advantage of CS concentrating the majority of the drug on the hair follicle disease lesions, ${ }^{8}$ thus making it highly effective, with fewer side effects. Although substantial research on the physicochemical and pharmaceutical properties of CS have been conducted, only a few studies have been conducted on their plasma and topical pharmacokinetics and in vivo efficacy as well as specific topical applications. ${ }^{9}$

Cryptotanshinone (CTS), a lipophilic compound produced by Radix Salvia miltiorrhiza, shows strong activity in microcirculation improvement, inflammation resolution, skin lesion repair, and other aspects of treatment of skin diseases. ${ }^{10}$ Clinically, CTS has a remarkable effect in treating inflammatory and pustular acne. ${ }^{11}$ The major factors limiting the use of CTS are its low solubility in aqueous solvents, light instability, and induced effect on hepatic enzymes. In this context, considering the limitations of CTS and advantages of CS, we envisioned using CS as carriers of CTS to enhance the drug biocompatibility and prevent the undesirable side effects of the drug.

Recently, we have reported the in vitro skin-permeation behavior and in vivo microdialysis by skin-targeted delivery of paeonol from its microsponge formulation. ${ }^{12}$ Building on these precedents, we initiated a research program with the aim of evaluating the topical delivery system CTS-CS in terms of in vitro drug release, in vivo pharmacokinetics, and in vivo efficacy for topical therapy of acne. Herein, we report the first example

Received: September 8, 2016

Accepted: December 8, 2016

Published: December 20, 2016 



Figure 1. Transmission electron microscopy image of the formed CS (original magnification $40000 \times$ ).

of the preparation and assessment of a new topical delivery system for topical therapy of acne. In this article, a systematic study was conducted to investigate the in vitro and in vivo pharmacokinetics and pharmacodynamics of CTS-CS gel. To reflect the advantages of the CTS-CS formulation compared with the ordinary CTS formulation in permeation and drug retention, we studied the effects of the CS formulation on the drug by carrying out a skin-permeation test using a Franz diffusion cell. Meanwhile, the differences in the plasma and local pharmacokinetics and the in vivo efficacy of the two formulations were studied as well, to illustrate the advantages of CS in topical administration and their good application prospects in the treatment of skin diseases.

\section{RESULTS AND DISCUSSION}

Preparation of CTS-CS and Their Gel Formulation. The shape and morphology of CTS-CS on microscopic scales were investigated by transmission electron microscopy after negative staining, which are shown in Figure 1. The CS were spherical closed vesicles with a single-layer or multilayer phospholipid membrane outside and the drug packaged inside.

The loading efficiency was calculated according to eq 1 (see details in the Supporting Information). The encapsulation efficiency of the CTS-CS was $59.90 \pm 1.75 \%(n=3)$. The volume of the CTS-CS was determined from a particle size distribution map, which showed the particle size and distribution, determined by the laser light-scattering technique. The average particle size was $119.53 \pm 13.85 \mathrm{~nm}$ and the coefficient of dispersion was $0.25 \pm 0.025$. The dispersibility of the liposomes containing water-soluble ceramide was maintained for at least 2 months.

Previous experiments had been carried out for correlation with the formation of CTS-CS. A central composite rotatable design based on the response surface methodology was employed to design and formulate an appropriate CTS-CS formulation. Therefore, parameters such as the membrane material content, ratio of drug to lipid, and temperature were chosen as key variables to evaluate the effects on the loading efficiency and sorting coefficient. It has been well documented that the membrane material content determines the loading efficiency, whereas the membrane material content, ratio of drug to lipid, or correlation of the temperature and membrane material content determines the sorting coefficient.

CS with a high entrapping efficiency and small particle size could be prepared by the ethanol injection method, which is applied easily, and the formed CS showed a more stable morphology compared with those prepared by the filmingrehydration method, reverse evaporating method, or by other methods.

We had studied on the screening for the base of gel carbomer. ${ }^{12}$ The formed CTS-CS gel was smooth, uniform, had no obvious bubbles, and was easily applied, with appropriate stickiness. The measured content of CTS in the CS gel was $102.70 \mu \mathrm{g} / \mathrm{g}$, and in the ordinary gel, it was $102.54 \mu \mathrm{g} / \mathrm{g}$. During the experiment, the gel showed no deterioration, such as rancidity, stratification, or discoloration.

In Vitro Drug Release and Drug Deposition Studies of CS. Figure 2 shows the in vitro release profiles of CTS-CS gel,

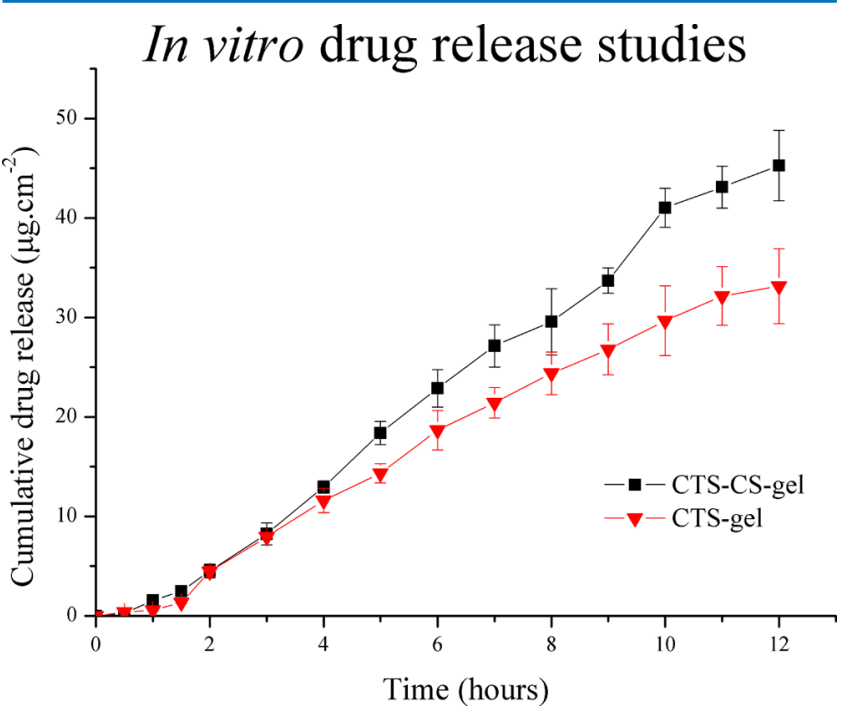

Figure 2. In vitro drug-release studies of CTS-CS gel $(0.01 \%, 1 \mathrm{~g})$ and CTS gel $(0.01 \%, 1 \mathrm{~g})$. All data are presented as means \pm SD $(n=3)$.

CTS gel, and a saturated aqueous solution. On the basis of the percutaneous cumulative penetration curves, the in vitro release profiles of the CTS-CS and CTS gels could be best explained by zero-order kinetics, as the cumulative drug release versus time was found to be linear $\left(R^{2}=0.994\right)$. The permeation rates of the CTS-CS and CTS gels were $4.0968 \pm 0.22$ and $3.0550 \pm$ $0.21 \mu \mathrm{g} / \mathrm{cm}^{2} \mathrm{~h}$, respectively. Compared with the CTS gel and saturated aqueous solution, the CS formulation could significantly increase the permeation rate of CTS. The curve of the CTS-CS gel showed sustained release for up to $12 \mathrm{~h}$. 
These advantages of drug release were probably attributed to the size $(119.53 \pm 13.85 \mathrm{~nm})$ and uniformity (dispersion coefficient, $0.25 \pm 0.025$ ) of the CS prepared by the ethanol injection method. Furthermore, the high affinity for keratin and moisturizing function of ceramide may be associated with the behavior of drug release with reduced transepidermal water loss/increased hydration of the SC, consequently leading to increased drug penetration into the skin. ${ }^{17,18}$

High-performance liquid chromatography (HPLC) analysis was used to determine the amount of CTS deposited in the skin from different formulations over different time intervals (Figure 3). As shown in Figure 3 and Table S4, the contents of

\section{Ex vivo drug deposition studies}

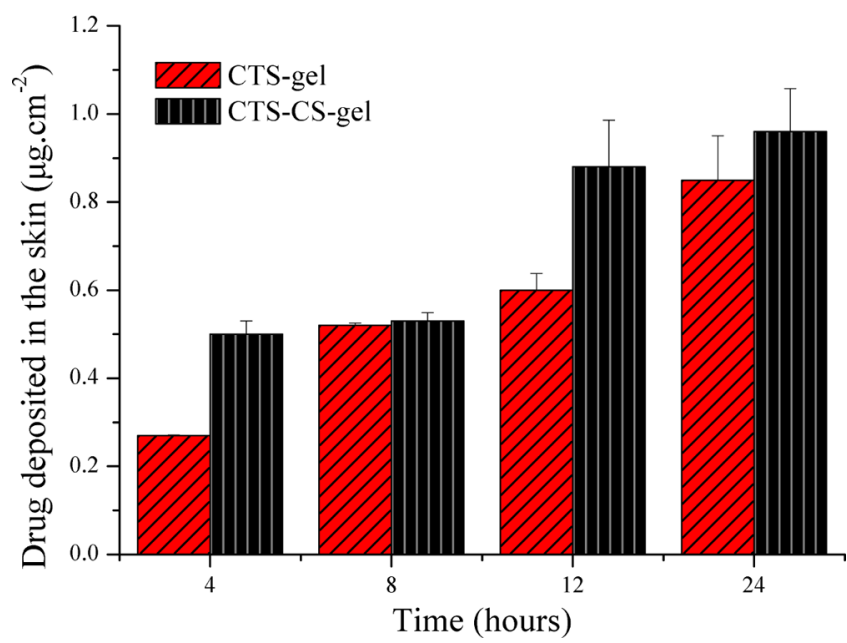

Figure 3. Ex vivo drug deposition studies of CTS-CS $(0.01 \%, 1 \mathrm{~g})$ and CTS $(0.01 \%, 1 \mathrm{~g})$ gels. All data are presented as means $\pm \operatorname{SD}(n=3)$.

CTS deposited in the skin at four time points was higher when the CTS-CS gel was applied compared to that when the CTS gel was applied, especially at $4 \mathrm{~h}(0.27 \pm 0.001$ vs $0.50 \pm 0.03$ $\left.\mu \mathrm{g} / \mathrm{cm}^{2}\right)$ and $12 \mathrm{~h}\left(0.60 \pm 0.038\right.$ vs $\left.0.88 \pm 0.106 \mu \mathrm{g} / \mathrm{cm}^{2}\right)$. The deposition of CTS from the CS gel at the same dose was the highest after $24 \mathrm{~h}$, showing that the CS gel improved drug delivery to the skin. Thus, the larger amount of drug deposition in the skin from the CTS-CS gel might be indicative of a greater efficacy in the topical area.

During the first $4 \mathrm{~h}$, the drug retention of the two preparations in skin was significantly different, indicating that the CTS-CS gel released a higher amount of the drug into the skin; however, their penetration rates were equivalent. From 4 $\mathrm{h}$ to $8 \mathrm{~h}$, the permeation rates of CTS-CS gel gradually increased which led to no significantly retention of drug in the skin compared to CTS gel. Over 8-12 h, the permeation rates of the CTS-CS gel continued to rise and there were significant differences in the skin retention of the drug, which were probably due to a drug reservoir formed by the CTS-CS gel in the skin until subsequent elimination. Nevertheless, the mechanism of drug release from CS is not clear.

It is known that skin permeability varies across animal species, and it is usually higher for animals with a high hairfollicle density. ${ }^{19}$ The in vitro permeation study through rat skin was consistent with the animal chosen in pharmacokinetics study for convenience operation of microdialysis and in vivo efficacy study for acne modeling.

Influence of CS on the Pharmacokinetics. In vivo recovery rates (RRs) were estimated according to the retrodialysis method, ${ }^{15}$ which relies on the assumption that diffusion through the semipermeable membrane is quantitatively equal in both directions. Thus, the compound of interest can be added to the perfusion medium and the disappearance rate through the membrane can be taken as the in vivo $R R$. The average recovery of CTS detected by the retrodialysis method was $51.83 \pm 1.68 \%(n=6)$ at the intradermal site and $51.52 \pm$ $0.97 \%(n=6)$ at the vein site. There was no significant statistical difference between the recoveries detected at the two sites of probe insertion $(P=0.734)$. The dialysis membrane showed a steady loss of CTS in the subcutaneous tissue fluid and in blood through the probe within the sampling time, confirming that recovery was consistent and reproducible.

The calibrated concentrations of free CTS intradermally and in the blood dialysate after topical treatment with CTS-CS and CTS gels over time are shown in Figure 4. The pharmacokinetic parameters estimated are reported in Table S5. Next, we compared the concentration-time profiles of the

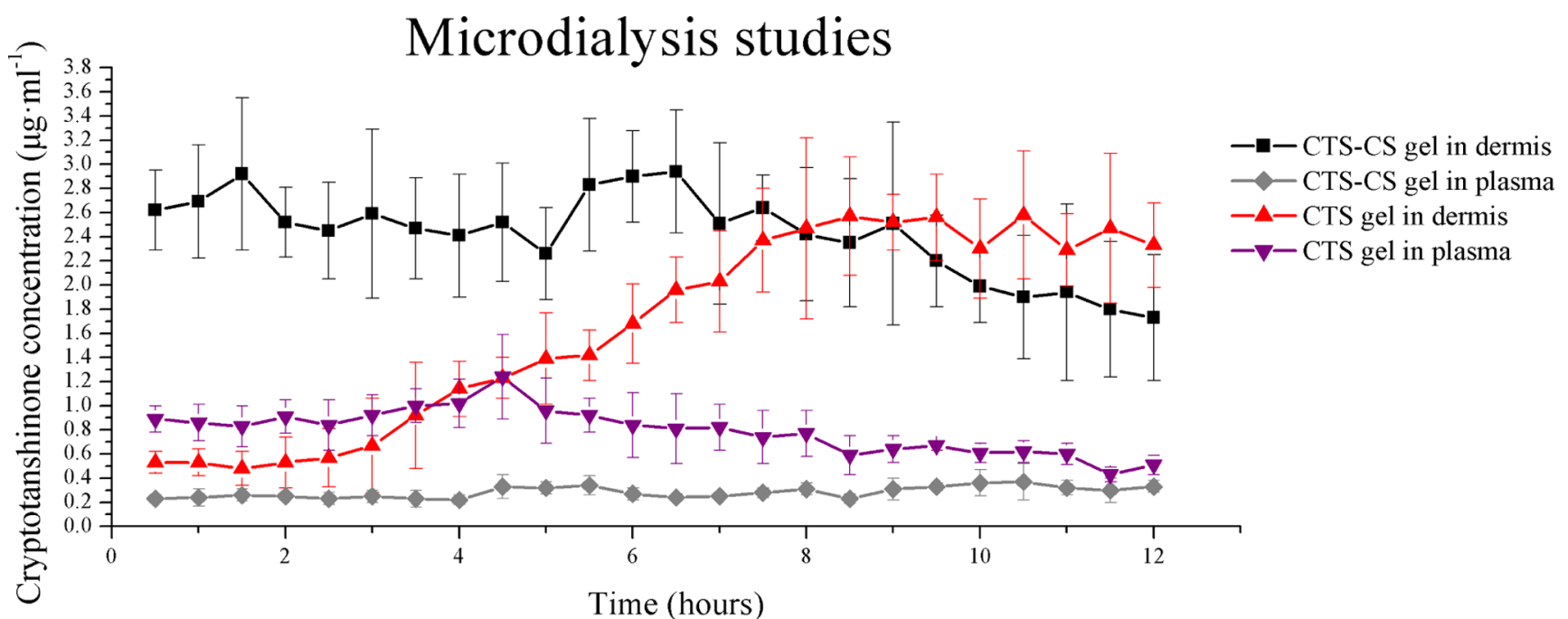

Figure 4. Concentrations of CTS in the intradermal liquid and plasma for the CTS-CS and CTS gels were detected by microdialysis. All data are presented as means $\pm \mathrm{SD}(n=3)$. 


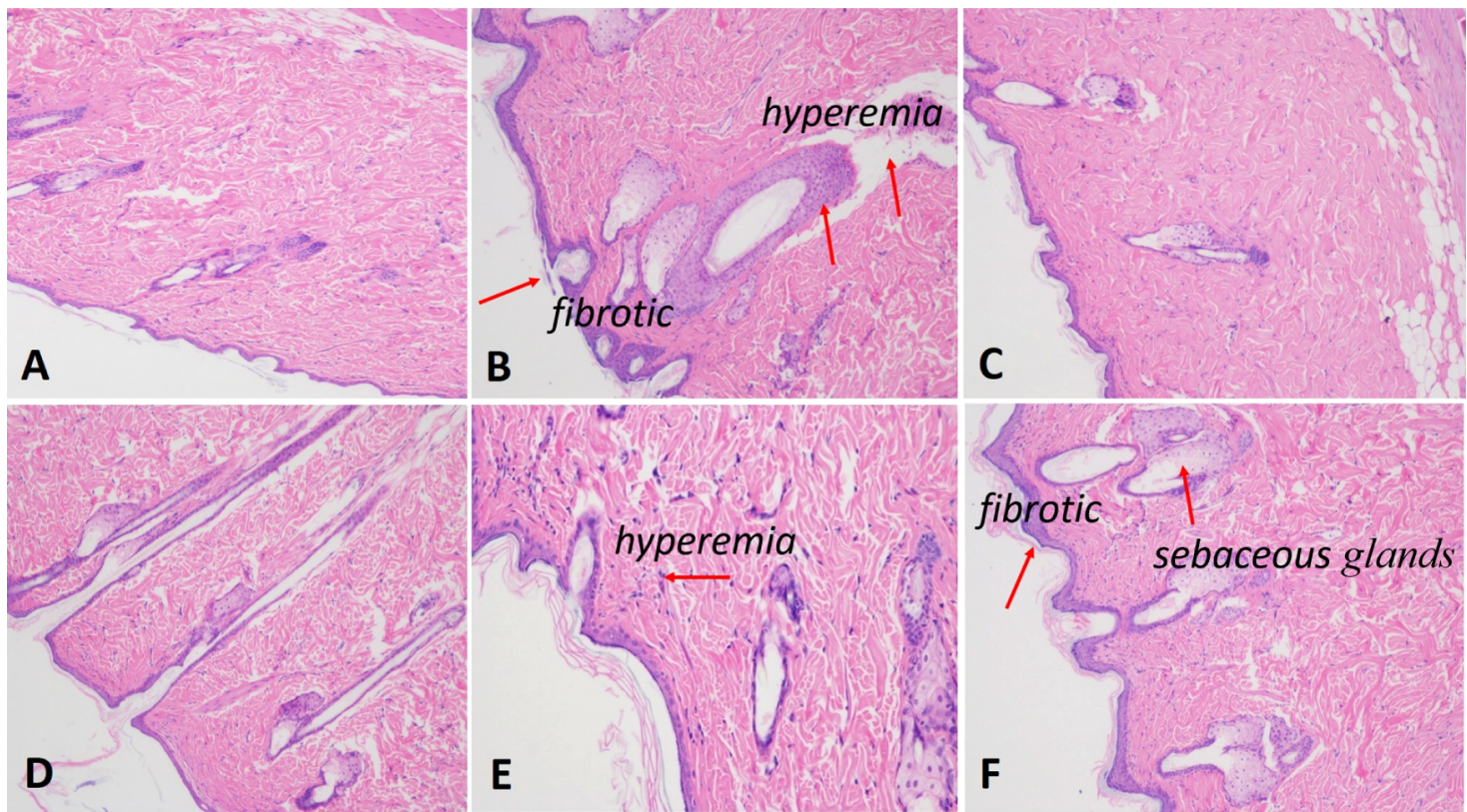

Figure 5. Hematoxylin-eosin (H\&E) staining of rat acne skin sections (100×). (A) Normal group, (B) model group, (C) positive control group, (D) CS group, (E) general group, and (F) blank matrix group. Arrows indicate pathological characteristics.

CTS-CS and CTS gels after dermal administration to rats. The area under the concentration-time curve $\left(\mathrm{AUC}_{0-t}\right)$, the time to peak concentration, and $t_{1 / 2}$ in the intradermal fluid for the CTS-CS gel were $27.99 \pm 0.89 \mu \mathrm{g} / \mathrm{mL} \mathrm{h}, 3.67 \mathrm{~h}$, and $9.90 \mathrm{~h}$, respectively, and for the CTS gel, they were $19.18 \pm 0.62 \mu \mathrm{g} /$ $\mathrm{mL} \mathrm{h}, 9.17 \mathrm{~h}$, and $5.44 \mathrm{~h}$, respectively. The $t_{1 / 2}$ value for the CTS-CS gel was twice that for the CTS gel, and there was a significant difference $(P<0.01)$ between the $\mathrm{AUC}_{0-t}$ values of the two preparations. The $\mathrm{AUC}_{0-t}$ and $C_{\max }$ values of the CTSCS gel in blood were less than half those of the CTS gel (3.31 \pm 0.27 vs $9.39 \pm 0.38 \mu \mathrm{g} / \mathrm{mL} \mathrm{h}$ and $0.45 \pm 0.09$ vs $1.36 \pm 0.19$ $\mu \mathrm{g} / \mathrm{mL}$, respectively), and all pharmacokinetic parameters of the two groups were significantly different $(P<0.05)$.

The CTS-CS gel had the following characteristics compared with the ordinary gel: a higher drug concentration, a shorter peak time, slower elimination, and less drug release into systemic circulation. The absorption rate of CTS was significantly higher on application of the CTS-CS gel compared to that on application of the CTS gel, as observed in Figure 4, which is in accordance with in vitro drug-release studies. The ability of CS to dissolve and penetrate into SC cells due to their unique lipid composition, which is similar to that of the SC, may lead to the faster penetration of CTS. A good distribution of the drug in the dermis allows the dose to be lowered and the formation of a drug reservoir within the upper layers of the SC, leading to concentration of the drug at the disease site, shown by the contrasting $\mathrm{AUC}_{0-t}$ values in the subcutaneous liquid. Likewise, slower elimination of CTS shows the capacity of the CS to release the drug slowly and maintain a stable epidermal residue concentration for a long time. ${ }^{19}$ Meanwhile, the lower measured values of $\mathrm{AUC}_{0-t}$ and $C_{\max }$ for CTS-CS gel in blood showed significant compared to CTS gel, resulted in reduced side effects.

Regarded as a dermal-delivery formulation, the drugs encapsulated in the CS should stay in the treated areas, and absorption to other areas should be minimized. In summary, in vivo pharmacokinetic studies show that the CTS-CS gel can actually improve the local bioavailability, reduce the side effects, and prolong the release time of the drug, which may lead to the retention of effective concentrations of the drug in the skin for a long time. The results show that preparing CTS as a CS formulation may be promising for treatment of skin diseases, like acne.

The low recoveries of drugs with large molecular weights, high lipophilicity, or high protein-binding rates limit the application of the microdialysis method. ${ }^{20}$ On the basis of previous research, ${ }^{12}$ microdialysis was proven to be an appropriate method for CTS (1.78 of oil-water partition coefficient, 166.18 of molecular weight, $46.16 \pm 9.02$ of proteinbinding rate). To determine the appropriate perfusate for CTS, the solubility of CTS in different solvents was studied. The solubility of CTS in distilled water was low, as well as in normal saline and phosphate-buffered saline (PBS) solution of different $\mathrm{pH}$, but $18.23 \mu \mathrm{g} / \mathrm{mL}$ in normal saline (containing $20 \%$ poly(ethylene glycol) (PEG)-400) which can be used as the perfusate of CTS.

There is controversy regarding the influence of the actual position of implantation of the probe on recovery and sample collection. Different depths of the probe in the dermis may influence the drug content in the intradermal fluid. At present, no appropriate instrumentation is available for measurement of the probe depth. Fartasch et al. have reported that if the probes are inserted by the same well-trained person, nearly consistent depths can be achieved. ${ }^{21}$ Simonsen et al. found that there was no significant correlation between the probe depth and drug concentration at a depth of $0.7-1.1 \mathrm{~mm} .^{22}$ In this study, the stainless needle was consistently inserted into the dermis by the same person in such a way that the needle was clearly visible through the superficial skin layer to make sure that the depth of the probe was not greater than $2 \mathrm{~mm}$, which corresponds to the depth of the deep dermis. ${ }^{23}$

In Vivo Efficacy of the CTS-CS Gel. Histology. Histological analyses were performed to assess the remission and improvement of symptoms of acne after treatment with the CTS-CS or CTS gel, compared to those in the positive control group and the blank matrix group. The results are present in 


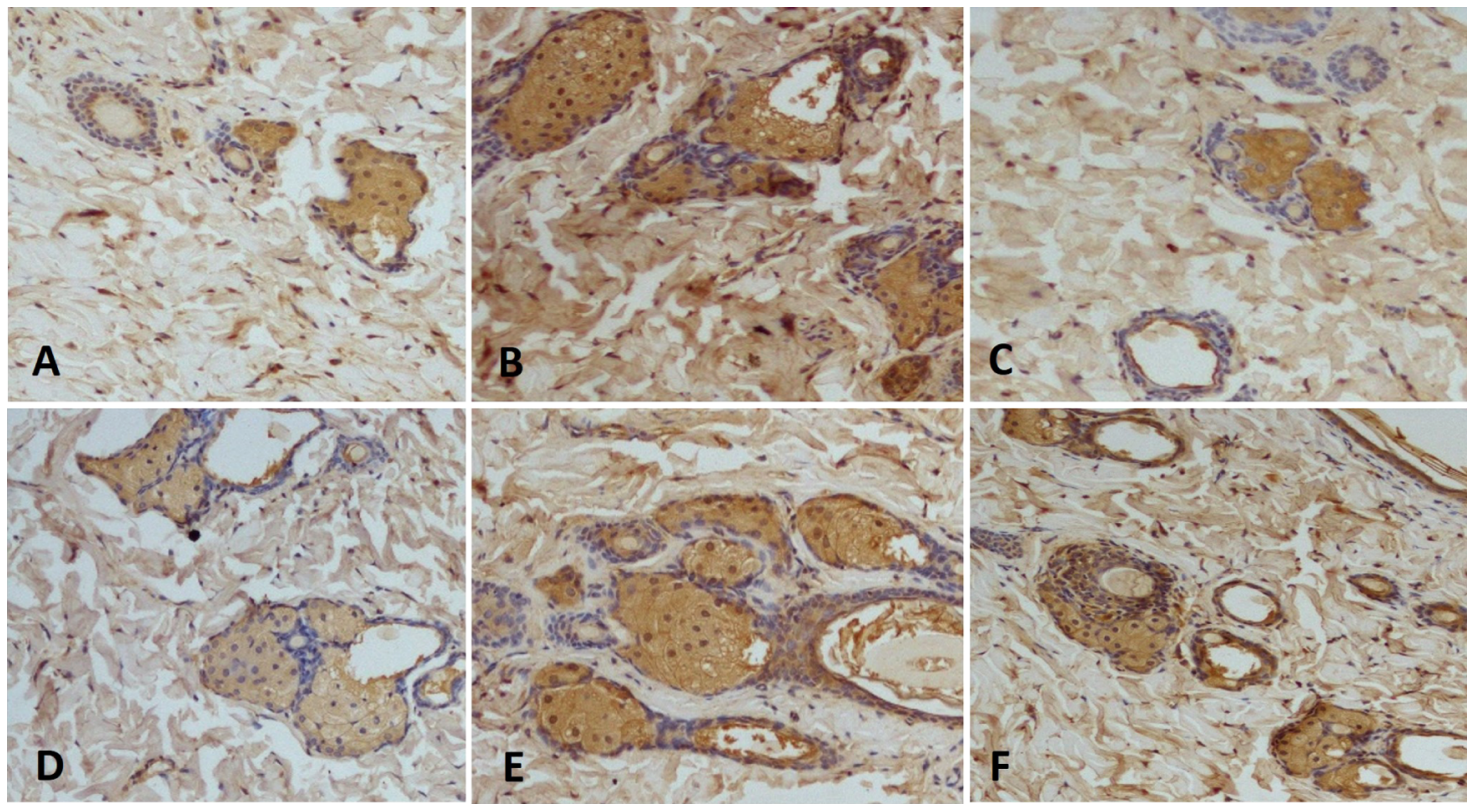

Figure 6. IL-1 $\alpha$ immunohistochemical staining of rat acne skin sections in the dermis (400X). (A) Normal group, (B) model group, (C) positive control group, (D) CS group, (E) general group, and (F) blank matrix group.
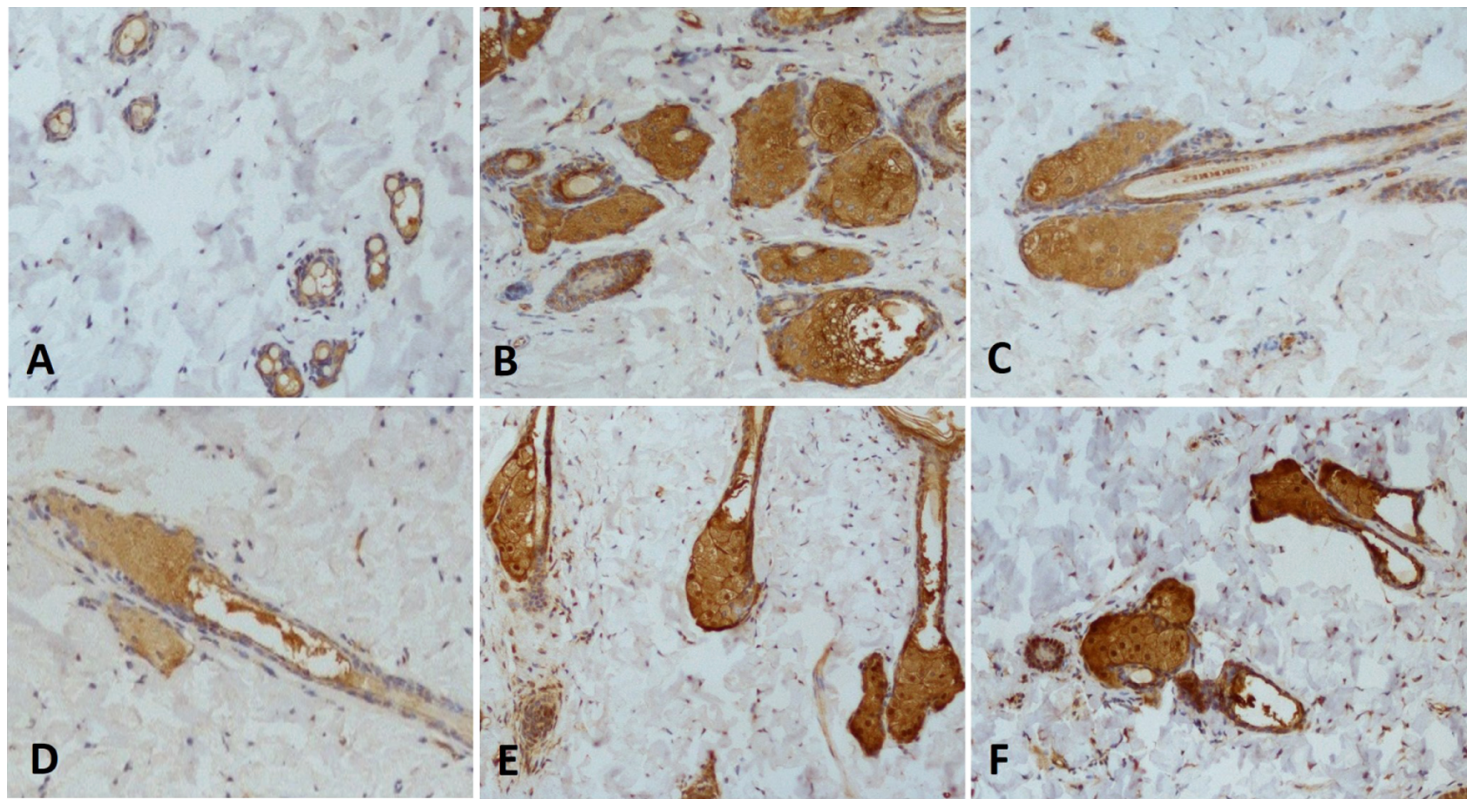

Figure 7. AR immunohistochemical staining of rat acne skin sections in the dermis (400X). (A) Normal group, (B) model group, (C) positive control group, (D) CS group, (E) general group, and (F) blank matrix group.

Figure 5. Compared with the normal group, the model group showed infiltration of inflammatory cells throughout the superficial dermis and excessive secretions from and a high density of distribution of the sebaceous glands; in addition, it showed hyperemia and tissue edema around the follicle roots. After dermal administration of the CTS-CS gel for 2 weeks, only very few inflammatory cells could be found in the CS group. The morphologies of the hair follicles and sebaceous glands returned to normal, that is, those in the positive control group. On treatment with the CTS gel, less secretion from and loose arrangement of the sebaceous glands were observed in the sectioned samples, but lots of inflammatory cells and congested pores still existed. There was little symptomatic improvement in the blank matrix group, indicating that the matrix had no effect on the results.

Immunohistochemistry (IHC). The observation and judgment of slice: positive expression of membrane and showing stained brown granules in the cytoplasm, the deeper the color, the stronger expression, no appearance of brown particles is negative. The average density quantization is summarized in Figures 6-8 and Table S6, compared with rats in the normal group, acne model rats showed a significant increase $(P<0.01)$ in the number of cells positive for interleukin (IL)- $1 \alpha$ and androgen receptors (ARs). Both the CTS-CS and CTS gels reduced the number of positive cells, but the CS and the positive control group showed a significant reduction $(P<$ 
A

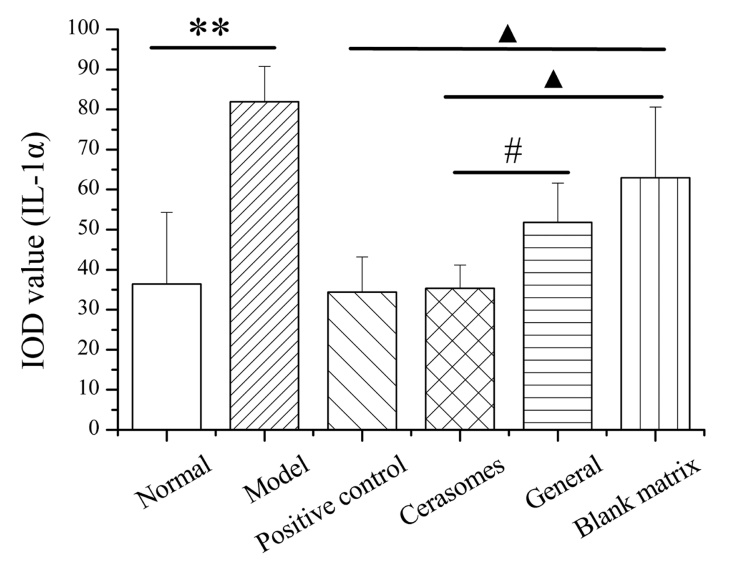

B

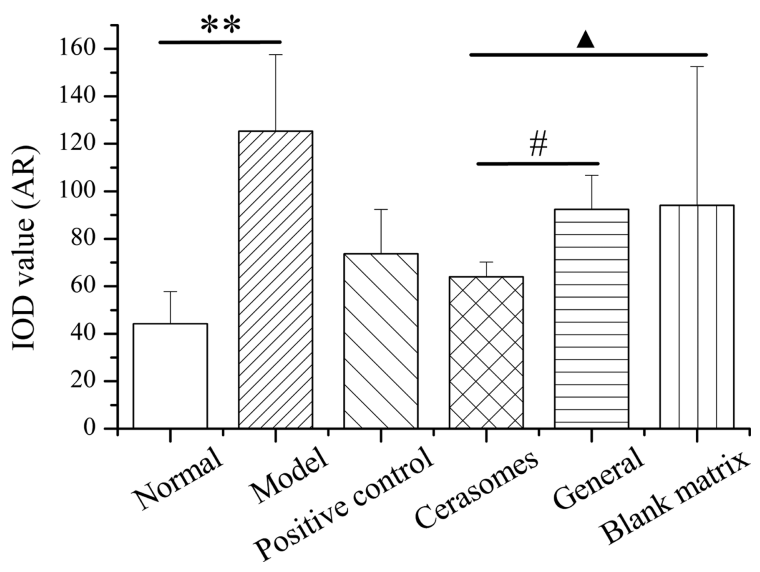

Figure 8. Positive expression of integrated optical density (IOD) in acne skin of rats: (A) IL- $1 \alpha$ and (B) AR. All data are shown as means \pm SD $(n=$ 6). ${ }^{* * P}<0.01$ vs normal group, $\boldsymbol{\Delta}_{P}<0.05$ vs blank matrix group, ${ }^{\#} P<0.05$ vs CS group.

\section{A}
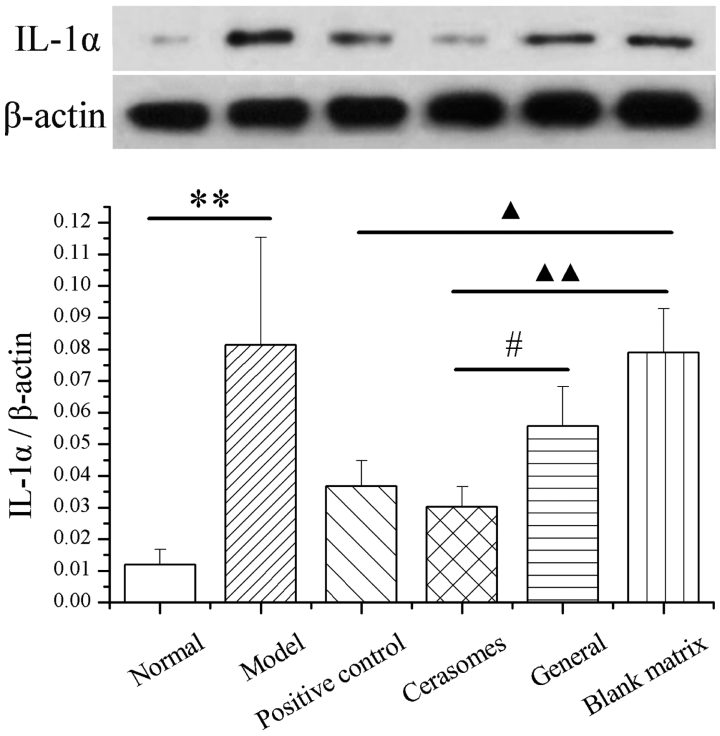

$\mathrm{B}$
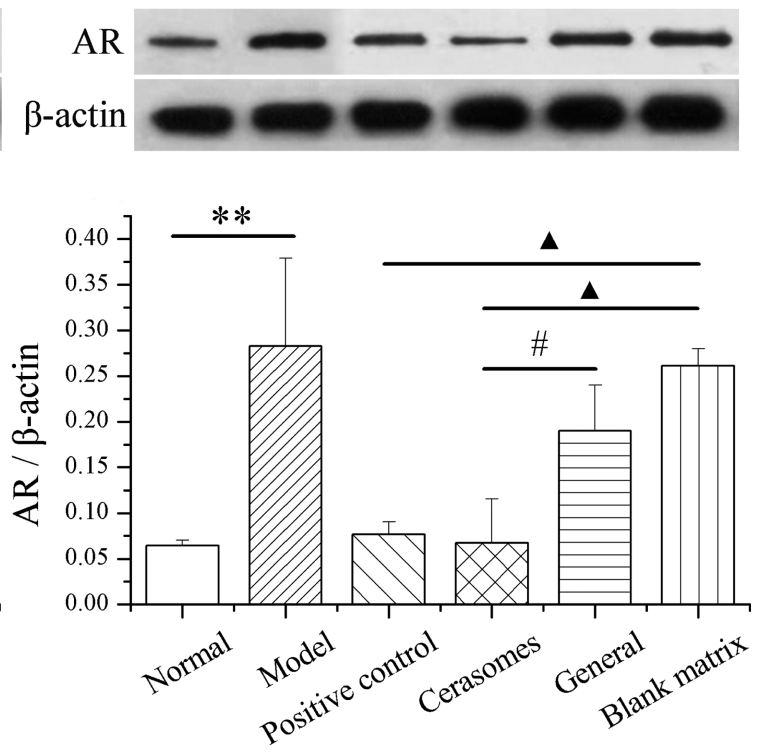

Figure 9. Expression of IL- $1 \alpha$ and AR measured by western blotting: (A) IL- $1 \alpha$ and (B) AR. All data are shown as means \pm SD ( $n=6$ ). **P< 0.01 vs normal group, $\boldsymbol{\Delta}_{P}<0.05$ vs blank matrix group, ${ }^{\#} P<0.05$ vs $C S$ group.

0.05) in the expression IL- $1 \alpha$, whereas only the CS group showed a significant decrease $(P<0.05)$ in AR expression.

Western Blotting for the Expression of $I L-1 \alpha$ and $A R$.

Western blotting analysis of the tissue extracts was conducted to directly detect the IL- $1 \alpha$ and AR contents in the rat skin. As shown in Figure 9, the IL- $1 \alpha$ and AR bands for the model group were apparently darker than those for the normal group. Compared with mice in the normal group, mice in the model group showed significant increases $(P<0.01)$ in the expression of IL- $1 \alpha$ and AR expressions in the skin. In the case of IL- $1 \alpha$, compared with the blank matrix group, the IOD of the CS group was the lowest compared to other three administrated groups, and had a significant decrease $(P<0.05)$ in contrast with the general group. The expression of the AR band showed the same trend among these groups, as shown in Figure 9 and Table S8.

The rat model of acne, induced by continuous smear of $100 \%$ oleic acid, was successfully prepared. The replication of animal model performances are shown in the following aspects: the thickening, hardening of local tissue in dorsal skin of rats, the red papules caused by ridges of hair follicle. From a subjective point of view, the amount of acne and roughness of the rat skin greatly improved after dermal administration of CTS-CS gel. Nevertheless, the differences among the six groups must be reflected in a more intuitive and subjective manner, such as through graphs and data. Hence, HE staining and immunohistochemical pathologies were used to observe the morphological changes in the rat epidermis and dermis and the positive expression of IL- $1 \alpha$ and ARs, respectively. In addition, western blotting was also adopted for semiquantitative analysis of the expression of IL- $1 \alpha$ and ARs.

The in vivo efficacy of the CTS-CS gel was characterized on the basis of the intensity of IL- $1 \alpha$ and AR positivity. IHC results showed that the model group of IL- $1 \alpha$ - and AR-positive cells increased significantly in the skin, suggesting that the stimulation of oleic acid can increase the density of expression of IL- $1 \alpha$ - and AR-positive cells in pilosebaceous unit, epidermis, and dermis. However, compared with the blank matrix group, 
the CS and drug-positive groups showed a significant decrease IL- $1 \alpha$ and AR positivity, which indicated that IL- $1 \alpha$ - and ARpositive cells can be inhibited by the CTS-CS gel and Kecuoyintong gel. Along with improvement of the symptoms of acne, variation in the density of IL- $1 \alpha$ - and AR-positive cells in back skin of rats may also have certain connections with the formation of acne. Western blotting detected that IL- $1 \alpha$ and AR positivity in the acne model group increased significantly compared to that in the blank group, whereas CS and the drugpositive group showed a significant decrease compared with the blank matrix group, showing that the CTS-CS gel and drugpositive group can restrain IL- $1 \alpha$ and AR expression to a certain extent, which is consistent with the immunohistochemical results.

Briefly, the studies found that the CTS-CS gel could relieve inflammation of the skin, as well as improve the degree of follicular keratosis and restore normalcy in the abnormally secreting sebaceous glands by regulating the expression of IL$1 \alpha$ and AR. The new preparation played a vital role in the treatment of acne vulgaris by inhibiting the stimulation of the male hormone in the sebaceous glands through competitive inhibition of ARs and by reducing inflammation of the skin through the expression of inflammatory cytokines. During animal grouping, we set up the blank matrix group for treatment with blank matrix gel, which showed no significant improvement after 2 weeks of administration, in contrast to the model group, revealing that the blank matrix in CTS-CS and ordinary gels had no effect on the final results.

In this research, IL- $1 \alpha$ and $\mathrm{AR}$ were chosen to be the pharmacodynamic indexes closely related to acne, as IL- $1 \alpha$ has been proven to be highly associated with excessive diversification of keratinocytes, induced by direct action on the IL-1 receptor or guiding the release of other growth factors, ${ }^{24,25}$ and the amount of AR determined the expression of androgen, which may cause sebaceous gland hyperplasia in the case of overexpression. ${ }^{26}$ From the viewpoint of CTS, it shows strong inflammation resolution and antiandrogenic activities. In summary, the above were fully illustrated the links between the two cytokines and the efficacy of CTS-CS gel indeed. Further studies are needed to elucidate the efficacy of the CTS-CS gel applied to acne lesions.

\section{CONCLUSIONS}

In this research, we put forward the first example of preparation and assessment of CTS-CS for topical therapy of acne. This study provided several potentially useful findings on the development of CS for topical treatment of skin diseases. First, a rapid drug permeation rate with a higher drug deposition is more effective in local treatment at a low dosage compared with ordinary formulations. Second, improved bioavailability in selected sites but minimum transdermal penetration into plasma led to fewer side effects for the same amount drug. Third, hair-follicle-targeting is very advantageous in curing hair-follicle diseases, like acne. These properties reveal that the CS dermal-delivery system could be applicable in pharmaceutical and cosmetic treatments. However, the exact mechanisms of drug distribution and drug penetration in the skin are still unknown. Besides, models combining the pharmacokinetic features of CS and pharmacodynamic index in therapy have not yet been evaluated. Further efforts will focus on in-depth assessment of CS and their formulation.

\section{MATERIALS AND METHODS}

Materials. The reference substance, CTS, was obtained from the National Institute for the Control of Pharmaceutical and Biological Products. PEG-400 was obtained from Tianjin Damao Chemical Reagent Factory, China. Normal saline containing 20\% PEG-400 was freshly prepared before use. Water-soluble ceramide was obtained from Xi'an Langhong Technology Co., Ltd., China. Soybean lecithin was purchased from Degussa Construction Chemicals AG, Europe. Cholesterol was purchased from Emieo-secoma Co. Oleic acid was supplied by Tianjin Fuyu Fine Chemical Co., Ltd., China. All other reagents were of analytical grade from Tianjin Damao Chemical Reagent Factory, China, and used as received.

Animals. Sprague Dawley (SD) rats (male and female, average body weight $180-220 \mathrm{~g}$, SPF grade) were purchased from the Experimental Animal Center in Guangzhou University of Chinese Medicine. All animals received care in compliance with the Care and Use of Laboratory Animals guidelines, and all procedures were conducted in accordance with the guidelines and ethical rules of the Experimental Animal Center in Guangzhou University of Chinese Medicine. All animals were allowed to adapt to the experimental conditions ( $12 \mathrm{~h}$ dark/ light cycle, temperature: $20 \pm 2{ }^{\circ} \mathrm{C}$, and humidity: $60 \pm 5 \%$ ) for 1 week. All surgeries were performed under $10 \%$ chloral hydrate anesthesia, and all efforts were made to minimize suffering.

Analytical System. A reverse-phase HPLC system, equipped with a quaternary pump (both model series 1260; Agilent Technologies Inc., Wilmington, DE), an autosampler, an online vacuum degasser, a column temperature controller, multiple-wavelength detectors and diode array, and an analytical workstation, was used for quantitative determination of CTS. Chromatographic measurements were conducted on an HPLC column, length $250 \mathrm{~mm}, 4.6 \mathrm{~mm}$ i.d., $5 \mathrm{~mm}$ particle size (Agilent $\mathrm{HC}-\mathrm{C}_{18}$; Agilent Technologies), using a mixture of acetonitrile and distilled water (80:20) as the eluent (flow rate: $1 \mathrm{~mL} / \mathrm{min}, 25^{\circ} \mathrm{C}$, wavelength: $276 \mathrm{~nm}$ ).

Preparation of CTS-CS. CTS-CS were prepared using the ethanol injection method, according to the procedure reported previously. ${ }^{13}$ To prepare the oil phase, CTS, cholesterol, and soybean lecithin were dissolved in $3 \mathrm{~mL}$ of anhydrous ethanol. Magnetic stirrers (C-MAG HS4, IKA, GER) were placed in the aqueous phase, which contained water-soluble ceramide dissolved in $10 \mathrm{~mL}$ of PBS $(0.2 \mathrm{M}, \mathrm{pH} 7.4)$, to warm it up gradually at a temperature of $55^{\circ} \mathrm{C}$, and stirring was performed with a stir bar at $600 \mathrm{rpm}$. Thereafter, the oil phase was gradually injected into the stirring aqueous phase with a syringe. Then, the mixture was stirred at $600 \mathrm{rpm}$ for $3 \mathrm{~h}$ at room temperature to allow the organic solvent to evaporate from the reaction flask. The resulting suspensions were harvested as we need and stored at $-5{ }^{\circ} \mathrm{C}$ prior to use. The optimized preparation was based on the loading efficiency and sorting coefficient (shown in the Supporting Information).

Preparation of CTS-CS Gel and the Skin Irritation Test. The gel matrix was composed of $2 \mathrm{~g}$ of carbomer, $15 \mathrm{~g}$ of propylene glycol, $20 \mathrm{~g}$ of glycerol, and water $(15 \mathrm{~mL})$; it was stirred at a slow stirring speed and had a swelling time of $5 \mathrm{~h}$. A CTS-CS or CTS ethanol solution was then added into 4 times the amount of blank matrix, while stirring the mixture with a magnetic stirrer to ensure a homogenous preparation, as well as dissolved sodium sulfate anhydrous $(0.2 \mathrm{~g})$ and sodium hydroxide $(0.2 \mathrm{~g})$ with water $(10 \mathrm{~mL})$, and finally added 
water up to $100 \mathrm{~g}$. As a comparison, we prepared a saturated solution of CTS using normal saline as a solvent. In our study, the content of CTS in the CS and ordinary gels were $102.7 \pm$ 0.67 and $102.54 \pm 1.27 \mu \mathrm{g} / \mathrm{g}$. The skin irritation test was carried out to evaluate the applicability of the two gels. The results showed that the two preparations caused no irritation to the skin in domestic rabbits (see details in the Supporting Information).

In Vitro Permeation and Drug Deposition Studies. In vitro drug permeation studies for CTS-CS or CTS ordinary gels were carried out using a Franz diffusion cell system, maintained at $32.0 \pm 1{ }^{\circ} \mathrm{C}$ by means of a circulating water bath. The receiver chamber of the diffusion cell $(15 \mathrm{~mL}$; orifice area of $3.14 \mathrm{~cm}^{2}$ ) was filled with receiver medium (20\% PEG-400normal saline, v/v) and stirred constantly at $300 \mathrm{rpm}$. Rat skin was used as a permeation membrane, which was obtained from female SD rats (average body weight $200 \mathrm{~g}$, SPF grade). The back skin was excised after the rat was euthanized, and the subcutaneous fat tissue was carefully removed with forceps; the samples were immediately stored at $-20{ }^{\circ} \mathrm{C}$ until analysis. The skin was clamped between the receptor and donor chambers, with the SC side facing upward. The respective formulation (CTS-CS gel, $1.0 \mathrm{~g}$; CTS gel, $1.0 \mathrm{~g}$; or saturated aqueous solution, $1 \mathrm{~mL}$ ) was gently applied to the SC side of the skin. The skin permeation membrane samples $(1 \mathrm{~mL})$ were withdrawn from the receiver compartment at 14 predetermined time points and analyzed for CTS concentration using a validated HPLC method, as described below. A cumulative curve of the total amount of CTS that permeated the membrane was plotted at each time interval versus time. The release kinetics and patterns of the CTS-CS and CTS gels were calculated or analyzed using different mathematical modes.

For quantification of the residual amount of drug in the skin, Franz diffusion cells were used as described above. The three formulations (1.0 g of CTS-CS gel, $1.0 \mathrm{~g}$ of CTS gel, and $1 \mathrm{~mL}$ of saturated aqueous solution) were administered in the donor chamber, and the skin samples were withdrawn at designated time points $(4,8,12$, and $24 \mathrm{~h})$. After removal of the remaining gel, the skin samples were weighed and flushed five times with $10 \mathrm{~mL}$ of distilled water. The remaining CTS in the rat skin samples was extracted by the tissue homogenate method with 5 $\mathrm{mL}$ of methanol. Ultimately, the tissue samples were centrifuged for $30 \mathrm{~min}$ at $10000 \mathrm{rpm}$ to obtained supernatants for HPLC analysis.

In Vivo Pharmacokinetics Study. Female SD rats weighing $180-220 \mathrm{~g}$ were anesthetized with a solution of $10 \%$ chloral hydrate administered intraperitoneally in a dose of $0.35 \mathrm{~mL} / 100 \mathrm{~g}$; thereafter, supplementary injections of half the dose were given every $90 \mathrm{~min}$ if required. The back fur of the rats was carefully shaved for intradermal microdialysis. Assembled by embedding a stainless steel needle into a tubing, an introducer was settled to implement intradermal insertion over the dermis. ${ }^{14}$ Once the needle was withdrawn, a microdialysis probe (CMA 20 Elite, $10 \mathrm{~mm}$ in membrane length, $20 \mathrm{kDa}$ molecular cut-off; CMA Microdialysis AB, Solna, Sweden) was inserted as a replacement, followed by removal of the tubing and taping it to the skin with surgical tape. Meanwhile, the jugular vein was isolated for plasma microdialysis, which was also implanted into a probe by means of the procedure described above. The probe was connected to a peristaltic micropump (CMA 402; CMA Microdialysis AB, Solna, Sweden), delivering the perfusion medium (20\% PEG$400-$ normal saline, $\mathrm{v} / \mathrm{v}$ ) at a constant flow rate of $2 \mu \mathrm{L} / \mathrm{min}$ after placement. After equilibration for $1 \mathrm{~h}, 1.0 \mathrm{~g}$ of the CTS-CS or CTS gel was applied to an area of back skin that was 3.14 $\mathrm{cm}^{2}$ in size. A refrigerated fraction collector (MAB 85; Microbiotech/se AB, Stockholm, Sweden) was used to collect the intradermal and plasma dialysates for HPLC analysis at 30 min intervals for $12 \mathrm{~h}$ from the same set animals at each time point.

Prior to the pharmacokinetic analysis, in vivo relative RRs of the probes were assessed according to the retrodialysis method $^{15}$ (shown in the Supporting Information). From the CTS concentrations calculated after calibration, four timeconcentration curves were constructed and certain pharmacokinetic parameters were determined, including the AUC, the maximum concentration reached $\left(C_{\max }\right)$, and the time necessary to reach the maximum concentration $\left(t_{\max }\right)$.

In Vivo Efficacy of the CTS-CS Gel. Animal Grouping. SD rats (half male and female; average body weight, $200 \mathrm{~g}$; SPF grade) were divided into the normal group, model group, positive control group, CS group, general group, and blank matrix group (six each) by the sortition randomization method.

Acne Model Replication. The back fur of the rats was shaved carefully with an electrical animal hair clipper. ${ }^{16}$ In addition to the normal group, $100 \%$ oleic acid $(0.5 \mathrm{~mL})$ was smeared onto the rat skin evenly over a depilating area of $4 \mathrm{~cm}^{2}$ once a day for 2 weeks. The normal group was treated with normal saline in the same volume.

Dermal Administration. When the acne model was successfully prepared, five of the groups received topical administration of Kecuoyintong gel, CTS-CS gel, CTS gel, and blank matrix gel $(0.5 / 100 \mathrm{~g}$, once a day) over the affected areas, excluding the model group.

After administration for 2 weeks consecutively, we observed and evaluated the macroscopic lesions of skin acne. Under anesthesia, all skin samples were cut at full thickness from the administration sites; they were then were used for histological analyses or frozen in liquid nitrogen immediately and maintained at $-80{ }^{\circ} \mathrm{C}$ for molecular and protein analyses. Thereafter, the animals were sacrificed by cervical dislocation.

Histology. The skin samples collected were fixed in $4 \%(\mathrm{w} /$ v) paraformaldehyde dissolved in PBS ( $\mathrm{pH} 7.4$ ), dehydrated with a graded series of ethanol, and embedded in paraffin. An RM2245 microtome (Leica Microsystems, Switzerland) was used to section the paraffin blocks into slices of $4 \mu \mathrm{m}$ thickness. Thereafter, the sectioned samples were treated in xylol to eliminate the paraffin, stained with $H \& E$, and finally visualized under an optical microscope (objective $100 \times$ ). Analysis of the pathological sections included study of the following markers relevant to acne: infiltration of superficial dermal inflammatory cells, hyperemia, and tissue edema around the follicle roots and sebaceous gland.

IHC. To study the behavior of acne during model replication and dermal administration, two markers related to acne, that is, IL- $1 \alpha$ and AR, were assessed for by IHC. They were quantified using IL- $1 \alpha$ and AR antibody detection kits following the manufacturer's instructions (shown in the Supporting Information). Images were acquired with an optical microscope (LV100D; Nikon, Japan) to observe positive staining, indicated by brown staining. The IOD and distribution of positive expression were measured using live imaging software, and they were then statistically analyzed.

Western Blotting Analysis. An anti-rat IL- $1 \alpha$ western blotting kit and an anti-rat AR western blotting kit were used to quantify the indexes, following the manufacturer's 
instructions (shown in the Supporting Information). An automatic image analytic system was then used to semiquantify the relative contents of the two pharmacodynamic indexes (the gray value of the image).

Statistical Analysis. Data are presented as means \pm SD. Statistical significance among the experimental groups was evaluated by one-way Student's $t$-tests or ANOVA. A $p$-value less than 0.05 was considered statistically significant, and a $p$ value less than 0.01 was considered highly significant.

\section{ASSOCIATED CONTENT}

\section{S Supporting Information}

The Supporting Information is available free of charge on the ACS Publications website at DOI: 10.1021/acsomega.6b00232.

Optimization of preparation technology; characterizations; skin irritation test; in vivo relative RRs of the probes; details of microdialysis experiments on rats; statistical analysis; release kinetics analysis; drug deposition studies; pharmacokinetics; IHC; western blotting analysis (PDF)

\section{AUTHOR INFORMATION}

\section{Corresponding Authors}

*E-mail: gzzhx2012@163.com (H.Z.).

*E-mail: gzlq2002@163.com (Q.L.).

ORCID

Qiang Liu: 0000-0002-9688-188X

\section{Author Contributions}

${ }$ T.Z. and H.C. contributed equally to this work.

Notes

The authors declare no competing financial interest.

\section{ACKNOWLEDGMENTS}

We sincerely thank the Natural Science Foundation of China (81373975), GuangDong College Chinese Cosmetics Engineering Centre Construction (GCZX-A1007), Guangdong Natural Science Foundation (2014A030310342), and the Youth Training Program of Southern Medical University (PY2013N009) for financial support.

\section{REFERENCES}

(1) (a) Katagiri, K.; Ariga, K.; Kikuchi, J. Preparation of organicinorganic hybrid vesicle "cerasome" derived from artificial lipid with alkoxysilyl head. Chem. Lett. 1999, 28, 661-662. (b) Liang, X.; Gao, J.; Jiang, L.; Luo, J.; Jing, L.; Li, X.; Jin, Y.; Dai, Z. Nanohybrid liposomal cerasomes with good physiological stability and rapid temperature responsiveness for high intensity focused ultrasound triggered local chemotherapy of cancer. ACS Nano 2015, 9, 1280-1293. (c) Jin, Y.; Li, Y.; Pan, H.; Dai, Z. Liposomal nanohybrid cerasomes for controlled insulin release. RSC Adv. 2014, 4, 42808-42815. (d) Jin, Y.; Pan, H.; Li, Y.; Dai, Z. Chitosan modified cerasomes incorporating poly (vinyl pyrrolidone) for oral insulin delivery. RSC Adv. 2014, 4, 5813758144. (e) Cao, Z.; Yue, X.; Li, X.; Dai, Z. Magnetic cerasomes for drug delivery. Langmuir 2013, 29, 14976-14983. (f) Liang, X.; Li, X.; Jing, L.; Xue, P.; Jiang, L.; Ren, Q.; Dai, Z. Design and Synthesis of Lipidic Organoalkoxysilane for Self-assembly of Liposomal Nanohybrid Cerasomes with Controlled Drug Release Properties. Chem. Eur. J. 2013, 19, 16113-16121. (g) Leung, S. L.; Zha, Z.; Teng, W.; Cohn, C.; Dai, Z.; Wu, X. Organic-inorganic nanovesicles for doxorubicin storage and release. Soft Matter 2012, 8, 5756-5764. (h) Cao, Z.; Yue, X.; Li, X.; Dai, Z. Cerasomal doxorubicin with longterm storage stability and controllable sustained release. Acta Biomater. 2012, 8, 3372-3380. (i) Cao, Z.; Yue, X.; Jin, Y.; Wu, X.; Dai, Z.
Modulation of Release of Paclitaxel from Composite Cerasomes. Colloids Surf., B 2012, 98, 97-104. (j) Liang, X.; Li, X.; Yue, X.; Dai, Z. Conjugation of porphyrin to nanohybrid cerasomes for photodynamic therapy of cancer. Angew. Chem., Int. Ed. 2011, 50, 11622-11627. (k) Ma, Y.; Dai, Z.; Zha, Z.; Gao, Y.; Yue, X. Selective Antileukemia effect of stabilized nanohybrid vesicles based on cholesteryl succinyl silane. Biomaterials 2011, 32, 9300-9307. (1) Ma, Y.; Dai, Z.; Gao, Y.; Cao, Z.; Zha, Z.; Yue, X.; Kikuchi, J. Liposomal architecture boosts biocompatibility of nanohybrid cerasomes. Nanotoxicology 2011, 5, 622-635. (m) Cao, Z.; Ma, Y.; Yue, X.; Li, S.; Dai, Z.; Kikuchi, J. Stabilized liposomal nanohybrid cerasomes for drug delivery applications. Chem. Commun. 2010, 46, 5265-5267.

(2) Katagiri, K.; Hamasaki, R.; Ariga, K. Layered paving of vesicular nanoparticles formed with cerasome as a bioinspired organic-inorganic hybrid. J. Am. Chem. Soc. 2002, 124, 7892-7893.

(3) Katagiri, K.; Hamasaki, R.; Ariga, K. Layer-by-layer selfassembling of liposomal nanohybrid "cerasome" on substrates. Langmuir 2002, 18, 6709-6711.

(4) Skiba-Lahiani, M.; Hallouard, F.; Mehenni, L.; Fessi, H.; Skiba, M. Development and characterization of oral liposomes of vegetal ceramide based amphotericin B having enhanced dry solubility and solubility. Mater. Sci. Eng., C 2015, 48, 145-149.

(5) Khan, N. R.; Harun, M. S.; Nawaz, A.; Harjoh, N.; Wong, T. W. Nanocarriers and their Actions to Improve Skin Permeability and Transdermal Drug Delivery. Curr. Pharm. Des. 2015, 21, 2848-2866.

(6) Coderch, L.; López, O.; de la Maza, A.; Parra, J. L. Ceramides and skin function. Am. J. Clin. Dermatol. 2003, 4, 107-109.

(7) Mojumdar, E. H.; Gooris, G. S.; Barlow, D. J.; Lawrence, M. J.; Deme, B.; Bouwstra, J. A. Skin lipids: localization of ceramide and Fatty Acid in the unit cell of the long periodicity phase. Biophys. J. 2015, 108, 2670-9.

(8) Peters, F.; Vorhagen, S.; Brodesser, S.; Jakobshagen, K.; Brüning, J. C.; Niessen, C. M.; Krönke, M. Ceramide synthase 4 regulates stem cell homeostasis and hair follicle cycling. J. Invest. Dermatol. 2015, 135, $1501-1509$.

(9) (a) Imura, T.; Sakai, H.; Yamauchi, H.; Kaise, C.; Kozawa, K.; Yokoyama, S.; Abe, M. Preparation of liposomes containing Ceramide 3 and their membrane characteristics. Colloids Surf., B 2001, 20, 1-8. (b) Yu, Z.; Lv, H.; Han, G.; Ma, K. Ethosomes Loaded with Cryptotanshinone for Acne Treatment through Topical Gel Formulation. PLoS One 2013, 11, No. e0159967.

(10) Jin, Y. C.; Kim, C. W.; Kim, Y. M.; Nizamutdinova, I. T.; Ha, Y. M.; Kim, H. J.; Seo, H. G.; Son, K. H.; Jeon, S. J.; Kang, S. S.; Kim, Y. S.; Kam, S. C.; Lee, J. H.; Chang, K. C. Cryptotanshinone, a lipophilic compound of Salvia miltiorrriza root, inhibits TNF-alpha-induced expression of adhesion molecules in HUVEC and attenuates rat myocardial ischemia/reperfusion injury in vivo. Eur. J. Pharmacol. 2009, 614, 91-97.

(11) Weckesser, S.; Engel, K.; Simon-Haarhaus, B.; Wittmer, A.; Pelz, K.; Schempp, C. M. Screening of plant extracts for antimicrobial activity against bacteria and yeasts with dermatological relevance. Phytomedicine 2007, 14, 508-516.

(12) Li, S. S.; Li, G. F.; Liu, L.; Jiang, X.; Zhang, B.; Liu, Z. G.; Li, X. L.; Weng, L. D.; Zuo, T.; Liu, Q. Evaluation of paeonol skin-target delivery from its microsponge formulation: in vitro skin permeation and in vivo microdialysis. PLoS One 2013, 8, No. e79881.

(13) Park, S. N.; Lee, M. H.; Kim, S. J.; Yu, E. R. Preparation of quercetin and rutin-loaded ceramide liposomes and drug-releasing effect in liposome-in-hydrogel complex system. Biochem. Biophys. Res. Commun. 2013, 435, 361-6.

(14) Stagni, G.; Donnell, D. O.; Liu, Y. J.; Kellogg, D. L.; Morgan, T.; Shepherd, A. M. Intradermal microdialysis: kinetics of iontophoretically delivered propranolol in forearm dermis. J. Controlled Release 2000, 63, 331-339.

(15) Sauernheimer, C.; Williams, K. M.; Brune, K.; Geisslinger, G. Application of microdialysis to the pharmacokinetics of analgesics: problems with reduction of dialysis efficiency in vivo. J. Pharmacol. Toxicol. Methods 1994, 32, 149-154. 
(16) Anigbogu, A.; Patil, S.; Singh, P.; Liu, P.; Dinh, S.; Maibach, H. An in vivo investigation of the rabbit skin responses to transdermal iontophoresis. Int. J. Pharm. 2000, 200, 195-206.

(17) de Jalon, E. G.; Blanco-Prieto, Ma. J.; Ygartua, P.; Santoyo, S. Topical application of acyclovir-loaded microparticles quantification of the drug in porcine skin layers. J. Controlled Release 2001, 75, 191197.

(18) Sakai, S.; Kikuchi, K.; Satoh, J.; Tagami, H.; Inoue, S. Functional properties of the stratum corneum in patients with diabetes mellitus: similarities to senile xerosis. Br. J. Dermatol. 2005, 153, 319-323.

(19) Chadawar, V.; Shaji, J. Microsponge Delivery System. Curr. Drug Delivery 2007, 4, 123-129.

(20) Incecayir, T.; Agabeyoglu, I.; Derici, U.; Sindel, S. Assessment of Topical Bioequivalence Using Dermal Microdialysis and Tape Stripping Methods. Pharm. Res. 2011, 28, 2165-2175.

(21) Schnetz, E.; Fartasch, M. Microdialysis for the evaluation of penetration through the human skin barrier-a promising tool for future research. Eur. J. Pharm. Sci. 2001, 12, 165-174.

(22) Simonsen, L.; Jørgensen, A.; Benfeldt, E.; Groth, L. Differentiated in vivo skin penetration of salicylic compounds in hairless rats measured by cutaneous microdialysis. Eur. J. Pharm. Sci. 2004, 21, 379-388.

(23) Stagni, G.; Shukla, C. Pharmacokinetics of methotrexate in rabbit skin and plasma after iv-bolus and iontophoretic administrations. J. Controlled Release 2003, 93, 283-292.

(24) Jugeau, S.; Tenaud, I.; Knol, A. C.; Jarrousse, V.; Quereux, G.; Khammari, A.; Dreno, B. Induction of toll-like receptors by Propionibacterium acnes. Br. J. Dermatol. 2005, 153, 1105-1113.

(25) Jeremy, A. H.; Holland, D. B.; Roberts, S. G.; Thomson, K. F.; Cunliffe, W. J. Inflammatory events are involved in acne lesion initiation. J. Invest. Dermatol. 2003, 121, 20-27.

(26) Alestas, T.; Ganceviciene, R.; Fimmel, S.; Müller-Decker, K.; Zouboulis, C. C. Enzymes involved in the biosynthesis of leukotriene B4 and prostagland in E2 are active in sebaceous glands. J. Mol. Med. 2006, 84, 75-87. 